\title{
Instituições de Ensino Superior e Especialização Inteligente: o contributo dos Institutos Superiores Politécnicos para a Implementação da RIS3 em Portugal
}

\author{
Mário Vale (a), Luís Balula (b), Luís Carvalho (c), José Manuel Simões (d) \\ (a) CEG, IGOT-Universidade de Lisboa, mario.vale@campus.ul.pt \\ (b) CEG, IGOT-Universidade de Lisboa, luis.d.balula@gmail.com \\ (c) CEGOT, Faculdade de Letras-Universidade do Porto, Icarvalho@letras.up.pt \\ (d) CEG, IGOT-Universidade de Lisboa, jmsimoes@campus.ul.pt
}

\begin{abstract}
Resumo
O desenvolvimento de um sistema de inovação não dispensa as instituições de ensino superior (IES), cujas funções se aproximam progressivamente do modelo triple helix (Etzkowitz e Leydesdorff, 2000). Apesar de tudo, os benefícios do investimento na ciência e tecnologia fazem-se sentir lentamente nas regiões periféricas, ainda que as IES se afirmem como um ator primordial nas estratégias de desenvolvimento das regiões periféricas (Goddard et al, 2012). Esta comunicação discute o contributo dos institutos politécnicos para a concretização das prioridades da RIS3. A metodologia seguida assenta na análise de informação de fontes indiretas e diretas (inquérito) sobre os cursos, estudantes e graduados, publicações e projetos de investigação e contratos de transferência de tecnologia pertinentes em cada um dos politécnicos para a RIS3 da região onde se localizam. No final, formulam-se algumas sugestões para uma articulação mais profícua entre os politécnicos e a economia regional.
\end{abstract}

Palavras-chave: RIS3, Inovação, Institutos Superiores Politécnicos, Desenvolvimento Regional

\section{INTRODUÇÃO}

A política europeia tem vindo a promover ativamente o papel regional das Instituições de Ensino Superior (IES), quer através de uma Agenda para a Modernização do Ensino Superior que salienta a importância da economia do conhecimento para o desenvolvimento regional (EC, 2011), quer ao determinar que as estratégias de especialização inteligente (RIS3, ENEI, EREI) constituem um pré-requisito para o acesso a financiamento através dos fundos estruturais (EC, 2016). Destes dois fatores resulta um reforço claro da posição das IES regionais, que passam a ter, potencialmente, um papel ativo na definição e implementação de projetos e políticas regionais, assente em parcerias apoiadas por incentivos financeiros significativos, quer com agentes económicos privados, quer com entidades públicas.

Partindo de uma análise introdutória da literatura relevante sobre a importância das IES para o desenvolvimento regional, esta comunicação propõe uma metodologia para a avaliação do grau de alinhamento estratégico entre a ação dos Institutos Superior Politécnicos (ISP) e as Estratégias Regionais de Especialização Inteligente (RIS3/EREI) em Portugal.

Enquanto centros de conhecimento inseridos nas regiões, em contacto direto com as oportunidades, desafios e riscos das suas cidades e territórios, os ISP constituem parceiros incontornáveis nos processos de desenvolvimento regional. Dependendo do contexto local, das estruturas políticas e da liderança institucional, os ISP podem desempenhar seu papel de diferentes maneiras. No entanto, a sua ação será tanto mais eficaz quanto maior for o alinhamento entre as prioridades nos vários domínios de especialização RIS3/EREI da região e a estra-tégia de produção e difusão de conhecimento do ISP.

Neste sentido, esta comunicação desenvolve uma metodologia capaz de avaliar a adequação da ação regional dos ISP aos domínios prioritários da RIS3/EREI, em três dimensões críticas: ensino e formação; investigação e produção científica; transferência de conhecimento e tecnologia. A título de conclusão, formula-se um conjunto de sugestões para uma articulação mais profícua entre os politécnicos e a economia regional.

A avaliação contempla 13 ISP localizados em quatro regiões NUTS II distintas, nomeadamente: Norte 
(Bragança, Cávado e Ave e Viana do Castelo); Centro (Castelo Branco, Coimbra, Guarda, Leiria, Tomar e Viseu); Alentejo (Beja, Portalegre, Santarém); Área Metropolitana de Lisboa (Setúbal).

Ressalva-se que o objetivo do estudo não é o de estabelecer uma comparação competitiva entre os vários ISP, mas sim, antes do mais, o de fazer um diagnóstico geral propositivo, com vista a identificar os pontos fortes e as oportunidades estratégicas de cada ISP em função das especificidades da região que serve e onde se insere.

\section{ENSINO SUPERIOR, DESENVOLVIMENTO E COESÃO TERRITORIAL}

A relevância da ciência no progresso tecnológico tem sido evidente desde meados do séc. $X X$, constituindo uma fonte de conhecimento essencial para o crescimento económico centrado na inovação. A relação entre a ciência e tecnologia é indiscutível, sendo evidente a incorporação de conhecimento científico na inovação da oferta de bens e serviços, assim como na mudança de processos de organização da produção e do trabalho. Todavia esta relação é complexa, envolvendo múltiplos agentes e funcionando numa lógica de sistema de inovação, de âmbito nacional (Lundvall, 1988) e regional (Cooke, 1992).

O desenvolvimento de um sistema de inovação não dispensa as instituições de ensino superior. Tradicionalmente, as IES desempenha(ra)m funções de ensino e formação avançada e de investigação, evoluindo, mais recentemente, para um modelo triple helix (Etzkowitz e Leydesdorff, 2000), assente na articulação entre universidade - empresa - Estado. No entanto, esta 'terceira missão' nem sempre constitui um objetivo central nas IES.

O desenvolvimento das cidades e regiões ancorado em estratégias high-road implica apostas claras na inova -ção. Deve, no entanto, sublinhar-se que a era da inovação linear, top-down, conduzida por especialistas e orienta -da para a produção de bens e serviços está a perder terreno para diferentes formas e níveis de coprodução e coo -peração generalizada entre produtores, consumidores, clientes e cidadãos (Arnkil et al., 2010). Emergem novas formas de criação de conhecimento, que incluem a aprendizagem contínua, exploração, co-criação, experimentação, plataformas de consumidores e utilizadores, já designadas por quadruple ou quintuple helix (cultura e socieda -de civil) (Carayannis e Campbell, 2011). Trata-se, na verdade, de uma nova plataforma de inovação centrada nos cidadãos.

As IES têm privilegiado a formação de graduados (licenciados, mestres e doutores), apoiando deste modo o crescimento económico e o desenvolvimento social. Todavia, este papel tem vindo a ser complementado com a transferência de tecnologia e ligação à sociedade. As IES produzem conhecimento com potencial de valorização económica que pode ser transferido para os agentes económicos, através do estabelecimento de parcerias com empresas, que visam a transferência de tecnologia, do registo e licenciamento de propriedade intelectual (patentes), ou por via do empreendedorismo académico dirigido à comercialização direta do conhecimento (Goddard et al., 2012). Neste último caso, o empreendedorismo de origem académica está na base da génese de clusters em novas atividades intensivas em conhecimento.

Enquanto centros de produção de novo conhecimento, as IES potenciam knowledge spillovers (Jaffe, 1989), um tipo de externalidade do conhecimento que permite um aumento do ritmo de inovação dos agentes económi$\cos$ da região. Paralelamente, os spin-offs induzidos pelas universidades geram importantes benefícios económicos locais e regionais devido ao reforço das dinâmicas de clusterização em atividades inovadoras (Antonelli, 1999). Estas dinâmicas retroalimentam a mudança institucional nas IES, progressivamente mais focadas na criação de estruturas de transferência de tecnologia, no apoio ao empreendedorismo académico e na criação de programas interdisciplinares de formação e investigação alinhados com as prioridades económicas nacionais e regionais.

A relação entre a formação e a investigação científica e inovação é imprescindível na economia do conhecimento, onde as IES desempenham um papel central no crescimento das atividades inovadoras e contribuem ativamente para o desenvolvimento da agenda social e ambiental da cidades e regiões. Esta inter-relação é tão ou mais pertinente nas regiões menos desenvolvidas, atendendo a que o crescimento económico alavancado por atividades inovadoras depende, em grande medida, de externalidades do conhecimento gerado pela academia, conhecidas que são as debilidades de muitas empresas e instituições destas regiões.

Os benefícios do investimento em Investigação e Desenvolvimento (I\&D) não são imediatos para a economia e talvez ainda sejam mais lentos nas regiões periféricas, precisamente onde é mais pertinente o investimento em ciência e tecnologia. Este paradoxo da inovação regional decorre da aparente contradição entre a maior necessidade de investir em inovação nas regiões periféricas e menor capacidade de absorção de fundos públicos para apoiar o investimento em atividades inovadoras (Oughton et al., 2002) ou de implementação de aplicações resultantes da investigação realizada pelas universidades da região (Christopherson e Clark, 2010). 
A influência das IES nas políticas territoriais é concomitante com o abandono das orientações de política regional que favoreciam exclusivamente a atração de investimento em favor da capacitação institucional e do place-based development (Barca Report). Neste sentido, as IES afirmam-se como um elemento primordial nas estratégias de desenvolvimento territorial, o que se tem traduzido por uma maior integração entre as políticas de ciência e as políticas de desenvolvimento regional (Goddard et al., 2012).

A relevância das IES para as regiões não se resume à inovação e ao desenvolvimento económico. Com a emergência de perspetivas mais holísticas de desenvolvimento, abarcando as esferas da equidade social, sustentabilidade ambiental, bem-estar, dinâmica cultural, reconfigura-se o envolvimento das IES na cidade e região. Ainda que em fase embrionária em algumas IES, diferentes elementos da academia desenvolvem investigação e estabelecem ligações com as comunidades locais em temáticas que extravasam, embora contribuam para, o desenvolvimento económico, tais como a inovação social, a cultura e as artes (Goddard e Vallance, 2013).

O sistema de ciência e tecnologia em Portugal evoluiu muito favoravelmente ao longo dos últimos anos em resultado da prioridade atribuída à política de I\&D e ao apoio decisivo dos Fundos Estruturais ${ }^{1}$. Com a publicação do RJIES em 2007, consagra-se o sistema binário de ensino superior, formado pelo ensino universitário e pelo ensino politécnico, com objetivos diferenciados: aquele de índole mais teórica e científica e este de cariz fundamentalmente mais prático e experimental. Os Institutos Superiores Politécnicos (ISP) públicos cobrem amplamente o território nacional, diferenciando-se o seu padrão geográfico pela presença em áreas menos desenvolvidas do país.

A ligação dos ISP ao tecido económico e social das regiões de acolhimento é determinante para o desenvolvimento e coesão territorial do país. Atendendo à especificidade da missão dos ISP, a sua inserção e contextualização territorial é preponderante nas estratégias e nas ações destas IES, tendo visto o seu papel reforçado com a agenda da RIS3 (EC, 2012).

A Estratégia de Investigação e Inovação para uma Especialização Inteligente preconiza o desenho e implementação de estratégias de desenvolvimento alinhadas com as vantagens específicas de cada região, quer sejam regiões avançadas ou periféricas, de alta ou média-baixa tecnologia, evitando o problema de one-size-fits-all patente em diversas políticas e estratégias de desenvolvimento. A racionalidade teórica desta abordagem assenta no conceito de path dependency do processo de desenvolvimento, historicamente contingente e específico de em cada lugar e região (Martin, 2010).

A capacidade institucional das regiões molda o sistema de inovação, com relevo para as instituições de ciência e tecnologia, empresas e organizações financeiras. A dinâmica de inovação assenta em processos de aprendizagem coletiva regional de natureza interativa e cumulativa. Pode afirmar-se que a geração de dinâmicas territorializadas de inovação requere não só empresas inovadoras como também instituições - em particular as IES preparadas para apoiar a troca e a exploração de diferentes tipos de conhecimento (básico/aplicado, genérico/ específico, tácito/codificado) (Tödtling, 1999).

As instituições de ciência e tecnologia, onde se destacam necessariamente os ISP pela sua vocação territorial, são um interface de articulação de redes globais e locais e desempenham um papel pivot na produção, difusão e recirculação do conhecimento entre os agentes económicos locais e regionais. São um ativo determinante para o desenvolvimento da base de conhecimento das empresas, para a qualificação do mercado de trabalho e para o desenvolvimento social e cultural das cidades e regiões.

\section{FUNDAMENTOS DE UMA PROPOSTA METODOLÓGICA PARA A AVALIAÇÃO DO ALINHAMENTO DOS ISP COM AS PRIORIDADES DAS EREI/RIS3}

Em conformidade com o novo papel das IES no desenvolvimento das regiões, a estratégia metodológica para a avaliação dos ISP deve incidir nas inter-relações que se estabelecem entre as três principais funções que estes desempenham, nomeadamente o ensino, a investigação e relação com a comunidade e com a sociedade em geral. A Figura 1 exemplifica as múltiplas relações entre ensino e investigação (integração de atividades na academia), ensino e envolvimento social e comunitário (participação alargada entre academia e sociedade) e investigação e envolvimento social e comunitário (impacto socioeconómico).

As novas funções das IES extravasam o ensino e a investigação, não se esgotando, porém, na transferência

${ }_{1}^{1}$ Um diagnóstico recente do sistema nacional de investigação científica pode ser consultado em Diagnóstico do Sistema de Investigação e Inovação: Desafios, Forças e Fraquezas Rumo a 2020, FCT, Lisboa, 2013. 
tecnológica e apoio à atividade económica e comercialização de tecnologia. Breznitz e Feldman (2012) mencionam um conjunto diverso de novos papéis desempenhados pelas IES, designadamente nas vertentes da iniciativa económica e das políticas públicas.

O diagrama da Figura 2 é ilustrativo da forma como a cooperação institucional entre um ISP e os ato-res locais e regionais influentes nos processos de governança, com particular destaque para os diversos serviços da administração pública (entidades municipais, CIM, CCDR, ou os serviços desconcertados do Estado nas áreas da saúde, segurança social, emprego e formação profissional), pode contribuir para a otimização de um sistema de inovação regional competitivo, capaz de projetar a região no exterior, posicionando-a, eventualmente, na economia global.

A estratégia de desenvolvimento das regiões está vinculada às estratégias regionais de especialização inteligente (EREI ou RIS3). O conceito de especialização inteligente refere-se a uma abordagem baseada na especificidade dos lugares e caracteriza-se pela identificação de áreas estratégicas de intervenção capazes de influenciar a especialização económica, científica e tecnológica de uma região e, consequentemente, a sua produtividade, competitividade e trajetória de crescimento económico (OECD, 2013). Estas estratégias foram definidas no âmbito da preparação da programação do Portugal 2020, seguindo orientações específicas da UE. As estratégias foram desenhadas levando em linha de conta a evolução da especialização económica das regiões e os objetivos dos agentes económicos e dos atores regionais, procurando evitar a seleção a priori de setores/atividades económicas sem que se fizesse uma demonstração do seu real potencial de crescimento.

A evolução tecnológica das regiões segue determinadas trajetórias decorrentes de opções estratégicas e das competências dos atores. As IES - tanto as Universidades como os Politécnicos - são atores cruciais do sistema de ciência, tecnologia e inovação e contribuem para a melhoria das qualificações e para a inovação tecnológica as regiões e do país. É, portanto, relevante avaliar o contributo dos ISP para a concretização das EREI, numa trajetória de evolução tecnológica regional.

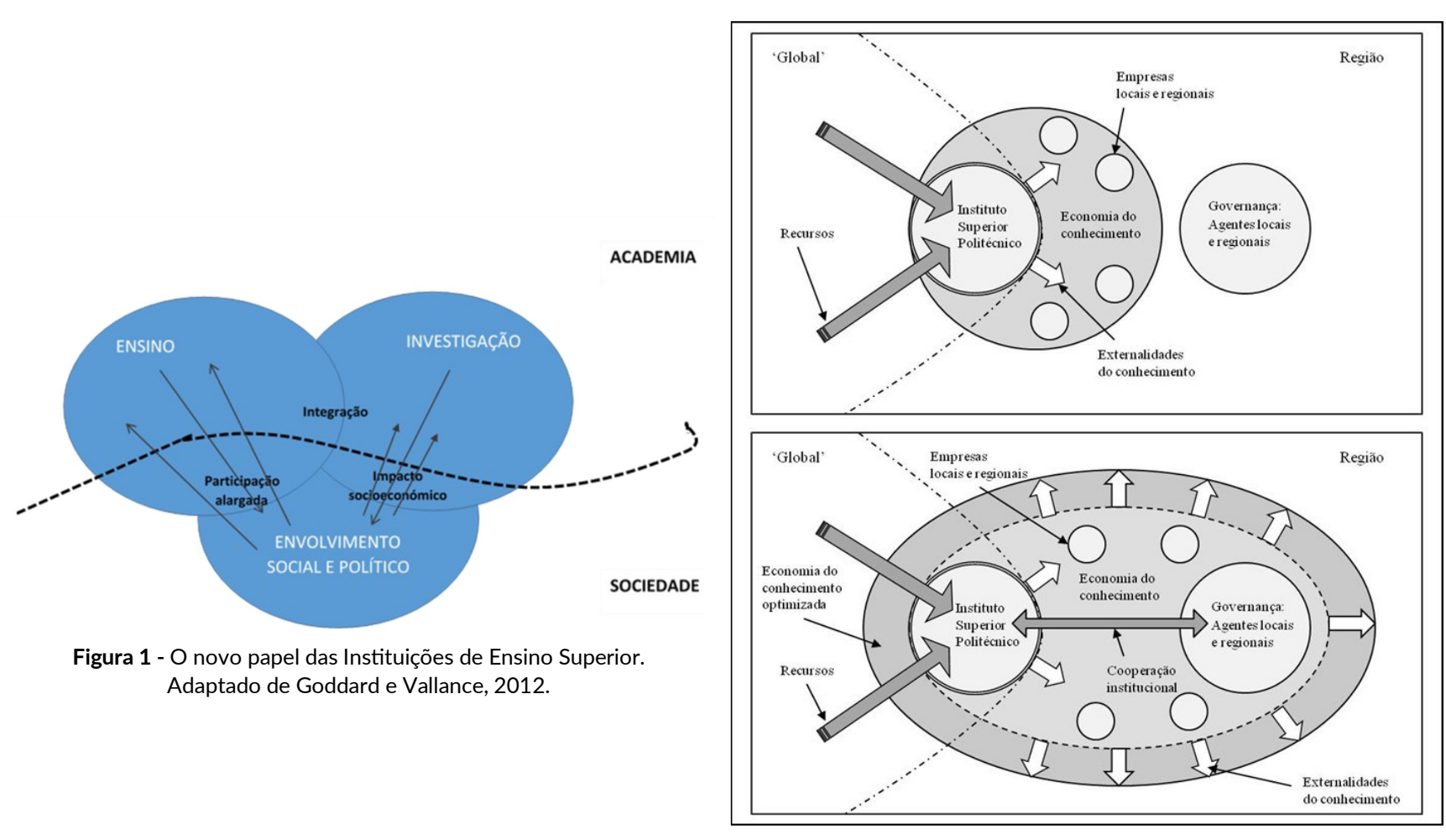

Figura 2. Importância da cooperação institucional entre o ISP e os agentes de governança local/regional para a otimização de um sistema de inovação regional competitivo. Adaptado de Benneworth \& Hospers (2006). 


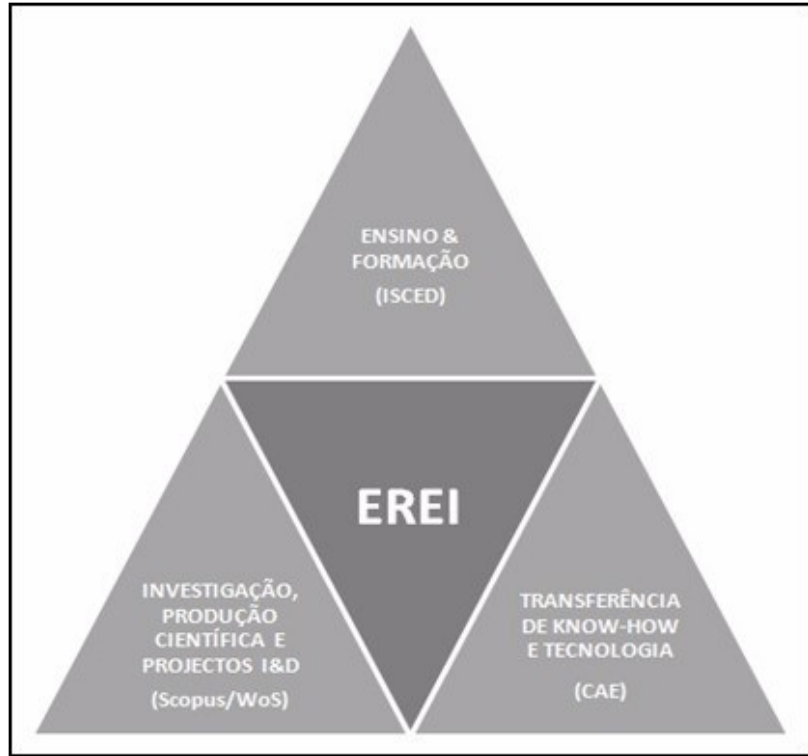

Figura 3 - Principais dimensões analíticas do estudo.

\section{PROPOSTA METODOLÓGICA}

Para cada uma das três dimensões analíticas (ver Figura 3), apresenta-se o tipo de análise a realizar através de tabelas específicas a cada região, exemplificativas do trabalho a desenvolver. Em primeiro lugar, para cada domínio de especialização da EREI (ou RIS3) respetiva, identificam-se as áreas de ensino e formação mais relevantes em cada ISP. Esta distribuição é mutuamente exclusiva; quer dizer, ainda que a mesma área educativa possa ser relevante para mais que um domínio, opta-se por atribuí-la ao domínio mais relevante, de acordo com o racional apresentado para cada EREI. Adota-se, para o efeito, a classificação internacional das áreas educativas do ensino superior consagrada pela UNESCO no International Standard Classification of Education (ISCED, 2013).

O confronto das áreas educativas identificadas com a oferta de cursos em cada ISP, com base

nos dados publicados pela Direção Geral de Estatísticas do Ensino Superior, permite-nos avaliar: (i) as áreas fortes e fracas da oferta educativa do ISP em função da respetiva EREI; (ii) o tipo e o número dos cursos oferecidos que correspondem às prioridades da EREI; (iii) a evolução recente do número de alunos que frequentam esses cursos.

Em segundo lugar, avalia-se a adequação das atividades de investigação e de produção científica dos ISP aos domínios de especialização da EREI. Para isso, recorrendo às bases de dados Scopus e/ou WoS, identificam-se, igualmente de forma mutuamente exclusiva, as áreas de publicação científica relevantes para cada domínio de especialização da EREI. Desta forma, quantificando o número de artigos publicados nos últimos cinco anos e referenciados nas bases Scopus/WoS para cada área de publicação, é possível identificar as atuais áreas dinâmicas do ISP, em termos de produção científica, e compará-las com a respetiva estratégia regional. Considera-se igualmente o envolvimento dos ISP em projetos científicos com financiamento competitivo aos níveis nacional (designadamente os financiados pela FCT e outras instituições de apoio à atividade científica) e internacional (H2020, EEA Grants, ESPON, etc.), verificando-se o seu alinhamento com as prioridades definidas para a estratégia de desenvolvimento da região.

Finalmente, quanto à transferência de know-how e tecnologia pretende-se analisar a relação entre a EREI e a dinâmica do ISP junto às empresas. A partir dos dados a obter junto aos serviços dos ISP, e recorrendo à classificação de atividades económicas (divisões da CAE) será possível determinar o grau de especialização (ou de diversificação) do ISP nas suas relações com o tecido empresarial, nas várias dimensões estratégicas da EREI. Paralelamente, uma análise das tipologias dos projetos apoiados pelo Programa Compete e pelos Programas Operacionais Regionais permitirá identificar o modo como as EREI estão a ser operacionalizadas em cada uma das regiões contribuindo para a validação dos resultados da nossa análise.

Apresentam-se, os instrumentos-tipo de organização da informação nas três dimensões indicadas. No primeiro caso (Ensino e formação) identificam-se as áreas de ensino e formação (ISCED) consideradas relevantes para cada um dos domínios de especialização da respetiva EREI. Para as outras duas dimensões (Investigação e produção científica; Transferência de know-how e tecnologia) apresentam-se apenas as tabelas-base onde se identificam, tal como no primeiro caso, para cada domínio estratégico, nomeadamente: (i) as áreas de publicação científica mais relevantes para as prioridades EREI (a partir das categorias das bases de dados Scopus e/ou WoS); (ii) os projetos de investigação científica em que o ISP esteve ou está envolvido (iii) os projectos apoiados no âmbito do Plano Operacional Regional e as empresas com as quais o ISP contratou prestações de serviços nos últimos 5 anos (a partir das divisões da CAE destas actividades económicas). 
Tabela I - Educação e formação: Análise EREI/ISCED (exemplo região Norte).

\begin{tabular}{|c|c|c|c|c|c|c|}
\hline \multirow{2}{*}{$\begin{array}{l}\text { Domínios de } \\
\text { especialização - } \\
\text { Região NORTE }\end{array}$} & \multirow{2}{*}{$\begin{array}{l}\text { Áreas de Ensino e de Formação (ISCED 2013) relevantes } \\
\text { para as prioridades estratégicas }\end{array}$} & \multirow{2}{*}{$\begin{array}{l}N^{\circ} \text { de Cur- } \\
\text { sos ofereci- } \\
\text { dos entre } \\
2013 \text { e } 2016\end{array}$} & \multirow{2}{*}{$\begin{array}{l}N^{\circ} \text { de Cur- } \\
\text { sos ofereci- } \\
\text { dos em } \\
2015 / 16 \\
\end{array}$} & \multicolumn{3}{|c|}{$\mathrm{N}^{\circ}$ de Alunos por Curso } \\
\hline & & & & $2013 / 14$ & $2014 / 15$ & $2015 / 16$ \\
\hline \multirow{6}{*}{$\begin{array}{l}\text { Recursos do Mar } \\
\text { e Economia }\end{array}$} & 0522 Natural environments and wildlife & & & & & \\
\hline & 0712 Environmental protection technology & & & & & \\
\hline & 0713 Electricity and energy & & & & & \\
\hline & 0724 Mining and extraction & & & & & \\
\hline & 0732 Building and civil engineering & & & & & \\
\hline & 0831 Fisheries & & & & & \\
\hline \multirow{3}{*}{$\begin{array}{l}\text { Capital Humano e } \\
\text { Serviços Especia- } \\
\text { lizados }\end{array}$} & 0612 Database and network design and administration & & & & & \\
\hline & 0613 Software and applications development $\&$ analysis & & & & & \\
\hline & 0619 ICTs not elsewhere classified & & & & & \\
\hline \multirow{7}{*}{$\begin{array}{l}\text { Cultura, Criação e } \\
\text { Moda }\end{array}$} & 0211 Audio-visual techniques and media production & & & & & \\
\hline & 0212 Fashion, interior and industrial design & & & & & \\
\hline & 0213 Fine arts & & & & & \\
\hline & 0214 Handicrafts & & & & & \\
\hline & 0215 Music and performing arts & & & & & \\
\hline & 0723 Textiles (clothes, footwear and leather) & & & & & \\
\hline & 0731 Architecture and town planning & & & & & \\
\hline \multirow{4}{*}{$\begin{array}{l}\text { Indústrias da } \\
\text { Mobilidade e } \\
\text { Ambiente }\end{array}$} & 0714 Electronics and automation & & & & & \\
\hline & 0715 Mechanics and metal trades & & & & & \\
\hline & 0716 Motor vehicles, ships and aircraft & & & & & \\
\hline & 0722 Materials (glass, paper, plastic and wood) & & & & & \\
\hline \multirow{6}{*}{$\begin{array}{l}\text { Sistemas Agro- } \\
\text { ambientais e } \\
\text { Alimentação }\end{array}$} & 0511 Biology & & & & & \\
\hline & 0721 Food processing & & & & & \\
\hline & 0811 Crop and livestock production & & & & & \\
\hline & 0812 Horticulture & & & & & \\
\hline & 0821 Forestry & & & & & \\
\hline & 0841 Veterinary & & & & & \\
\hline \multirow{14}{*}{$\begin{array}{l}\text { Ciências da Vida e } \\
\text { Saúde }\end{array}$} & 0512 Biochemistry & & & & & \\
\hline & 0531 Chemistry & & & & & \\
\hline & 0911 Dental studies & & & & & \\
\hline & 0912 Medicine & & & & & \\
\hline & 0913 Nursing and midwifery & & & & & \\
\hline & 0914 Medical diagnostic and treatment technology & & & & & \\
\hline & 0915 Therapy and rehabilitation & & & & & \\
\hline & 0916 Pharmacy & & & & & \\
\hline & 0917 Traditional \& complementary medicine \& therapy & & & & & \\
\hline & 0921 Care of the elderly and of disabled adults & & & & & \\
\hline & 0922 Child care and youth services & & & & & \\
\hline & 0923 Social work and counseling & & & & & \\
\hline & 0929 Welfare not elsewhere classified & & & & & \\
\hline & 1022 Occupational health and safety & & & & & \\
\hline \multirow{5}{*}{$\begin{array}{l}\text { Capital Simbólico, } \\
\text { Tecnologias e } \\
\text { Serviços do Turis- } \\
\text { mo }\end{array}$} & 0231 Language acquisition & & & & & \\
\hline & 0414 Marketing and advertising & & & & & \\
\hline & 1013 Hotel, restaurants and catering & & & & & \\
\hline & 1014 Sports & & & & & \\
\hline & 1015 Travel, tourism and leisure & & & & & \\
\hline $\begin{array}{l}\text { Sistemas Avança- } \\
\text { dos de Produção }\end{array}$ & $\begin{array}{l}\text { Prioridade transversal a todas as estratégias. } \\
\text { As áreas educativas chave (TIC, Electrónica,Ciências infor- } \\
\text { máticas, Materiais, Metalurgia e Metalomecânica, etc.) } \\
\text { encontram-se contempladas nas outras prioridades estraté- } \\
\text { gicas }\end{array}$ & & & & & \\
\hline \multirow{8}{*}{$\begin{array}{l}\text { Áreas estratégicas } \\
\text { transversais }\end{array}$} & 0311 Economics & & & & & \\
\hline & 0411 Accounting and taxation & & & & & \\
\hline & 0412 Finance, banking and insurance & & & & & \\
\hline & 0413 Management and administration & & & & & \\
\hline & 0415 Secretarial and office work & & & & & \\
\hline & 0416 Wholesale and retail sales & & & & & \\
\hline & 0417 Work skills & & & & & \\
\hline & 0421 Law & & & & & \\
\hline
\end{tabular}


Instituições de Ensino Superior e Especialização Inteligente: o contribu-to dos Institutos Superiores Politécnicos para a Implementação da RIS3 em Portugal

Tabela II. Investigação e produção científica: Análise EREI/WoS/Scopus (exemplo região Norte).

\begin{tabular}{|l|l|l|}
\hline \multicolumn{1}{|c|}{ Domínios de especialização EREI - NORTE } & $\begin{array}{c}\text { Áreas de publicação (Scopus/WoS) } \\
\text { relevantes para as prioridades EREI }\end{array}$ & No de artigos referenciados $^{\circ}$ \\
\hline Recursos do Mar e Economia & & \\
\hline Capital Humano e Serviços Especializados & & \\
\hline Cultura, Criação e Moda & & \\
\hline Indústrias da Mobilidade e Ambiente & & \\
\hline Sistemas Agroambientais e Alimentação & & \\
\hline Ciências da Vida e Saúde & & \\
\hline Capital Simbólico, Tecnologias e Serviços do Turismo & & \\
\hline Sistemas Avançados de Produção & & \\
\hline
\end{tabular}

Tabela III. Investigação e produção científica: Análise EREI/Projetos de investigação científica (exemplo região Norte).

\begin{tabular}{|l|l|l|}
\hline \multicolumn{1}{|c|}{ Domínios de especialização EREI - NORTE } & $\begin{array}{c}\text { Projetos de investigação científica } \\
\text { nacionais (coordenação/ } \\
\text { participação) }\end{array}$ & $\begin{array}{c}\text { Projetos de investigação científica } \\
\text { internacionais (coordenação/ } \\
\text { participação) }\end{array}$ \\
\hline Recursos do Mar e Economia & & \\
\hline Capital Humano e Serviços Especializados & & \\
\hline Cultura, Criação e Moda & & \\
\hline Indústrias da Mobilidade e Ambiente & & \\
\hline Sistemas Agroambientais e Alimentação & & \\
\hline Ciências da Vida e Saúde & & \\
\hline Capital Simbólico, Tecnologias e Serviços do Turismo & & \\
\hline Sistemas Avançados de Produção & & \\
\hline
\end{tabular}

Tabela IV. Transferência de conhecimento e tecnologia: Análise EREI/ PO Compete, POR/CAE.

\begin{tabular}{|l|l|l|}
\hline \multicolumn{1}{|c|}{ Domínios de especialização EREI - NORTE } & $\begin{array}{c}\text { Divisões CAE dos projetos apoiados } \\
\text { no âmbito do Plano Operacional } \\
\text { Regional }\end{array}$ & $\begin{array}{c}\text { Divisões CAE das principais em- } \\
\text { presas com as quais o ISP realizou } \\
\text { contratos de prestação de serviços } \\
\text { nos últimos 5 anos }\end{array}$ \\
\hline Recursos do Mar e Economia & & \\
\hline Capital Humano e Serviços Especializados & & \\
\hline Cultura, Criação e Moda & & \\
\hline Indústrias da Mobilidade e Ambiente & & \\
\hline Sistemas Agroambientais e Alimentação & & \\
\hline Ciências da Vida e Saúde & & \\
\hline Capital Simbólico, Tecnologias e Serviços do Turismo & & \\
\hline Sistemas Avançados de Produção & & \\
\hline
\end{tabular}




\section{CONCLUSÃO E PRÓXIMOS PASSOS}

Esta comunicação apresentou uma proposta metodológica para aferir do contributo e alinhamento das estratégias dos ISP face às EREI dos seus territórios de referencia. Em jeito de conclusão, apresentam-se algumas questões e hipóteses de trabalho, a explorar em momentos subsequentes.

Institutos Superiores Politécnicos: até que ponto regionais? Dado o reforço recente de qualificações dos seus quadros, o aumento da produção científica e a prestação de serviços avançados à empresas, o trabalho responderá sobre até ponto as relações entre os ISP e a base económica é de cariz essencialmente subregional ou se, pelo contrario, se alarga a outras geografias.

- $\quad$ Para além de projetos de I\&D formais: o papel dos ISP no aumento da capacidade de absorção de tecnologia e competitividade das economias regionais. Com base na informação recolhida, a análise procurará responder sobre o tipo de contributos e relações dos ISP com a base económica por via i) da qualificação de pessoas e ii) oferta de serviços complementares que permitam às empresas subir e/ou manter competitividade nas cadeias de valor, aumentando a sua capacidade de participar em projetos de I\&D mais exigentes.

- $\quad$ Face às estruturas económicas regionais, que tipo de transformação estão os ISP a promover por via das suas atividades de I\&D e prestações de serviços? Reforço das especializações existentes, diversificação em sectores conexos, ou emergências de novas atividades?

- $\quad$ Face aos pontos anteriores, será feita uma reflexão sobre o contributo "revelado" dos ISP para as EREI e para a intensificação tecnológica e de conhecimento das suas regiões, nomeadamente em relação ao seu contributo "formal" (i.e. participação institucional na definição de uma estratégia regional).

\section{BIBLIOGRAFIA}

ANTONELLI, C. (1999). The evolution of the industrial organisation of the production of knowledge. Cambridge Journal of Economics, 23, 243-260.

ARNKIL, R., Järvensivu, A., Koski, P., \&Piirainen, T. (2010). Exploring the Quadruple Helix: Report of Quadruple Helix Research for the CLIQ Project. University of Tampere.

BARCA REPORT. (2009). An Agenda for a Reformed Cohesion Policy: A Place-based Approach to Meeting European Union Challenges and Expectations. Brussels.

BENNEWORTH, P. S. and HOSPERS, G. J. (2006), Urban competitiveness in the knowledge economy: universities as new planning animateurs, Cambridge to Consett, Working Paper. Newcastle upon Tyne: CURDS.

BREZNITZ, S., \& FELDMAN, M.P. (2012). The engaged university. J. Technol Transfer, 37, 139-157.

CARAYANNIS, E. G., \&CAMPBEL, D. F. J. (2011). Open innovation diplomacy and a 21st century Fractal Research, Education and Innovation (FREIE) Ecosystem: Building on the quadruple and quintuple helix innovation concepts and the "Mode 3" knowledge production system. Journal of Knowledge Economy, 2, 327-372.

CCDR-Alentejo (2014). Uma Estratégia de Especialização Inteligente para o Alentejo.

CCDR-Centro (2017). RIS3 do Centro 2020: Estratégia de Investigação e Inovação para uma Especialização Inteligente.

CCDR-LVT (2015). Especialização Inteligente de Lisboa 2014-2020.

CCDR-Norte (2014). Norte 2020: Estratégia Regional de Especialização Inteligente.

CHRISTOPHERSON, S., \& CLARK, J. (2010). Limits to the 'learning region': what university-centred economic development can (and cannot) do to create knowledge-based regional economies. Local Economy, 25(5), 120-30.

COOKE, P. (1992). Regional innovation systems: competitive regulation in the new Europe. Geoforum, 23(3), 365-382.

EC (2011). Supporting Growth and Jobs - an agenda for modernisation of Europe's higher education system. Com (2011) (567). CEC, Brussels.

EC (2012). Guide to Research and Innovation Strategies for Smart Specialisation (RIS3). European Commission.

ETZKOWITZ, H., \& LEYDESDORFF, L. (2000). The dynamics of innovation: from National Systems and "Mode 2" to a Triple Helix of university-industry-government relations. Research Policy, 29(2), 109-123.

GODDARD, J., ROBERTSON, D., \& VALLANCE, P. (2012). Universities, technology and innovation centres and regional development: The case of the North-East of England. Cambridge Journal of Economics, 36(3), 609-627.

GODDARD, J., \& VALLANCE, P. (2013). The university and the city. Abingdon: Routledge. 
JAFFE, A. B. (1989). Real effects of academic research. American Economic Review, 79, 957-970.

MARTIN, R. (2010). Roepke Lecture in Economic Geography-Rethinking Regional Path Dependence: Beyond Lock-in to Evolution. Economic Geography, 86(1), 1-27.

OECD (2013). Innovation-driven Growth in Regions: The Role of Smart Specialisation (preliminary version).

OUGHTON, C., LANDABASO, M., \& MORGAN, K. (2002). The regional innovation policy paradox: innovation policy and industrial policy. Journal of Technology Transfer, 27(1), 97-110.

TÖDTLING, F. (1999). Innovation networks, collective learning, and industrial policy in regions of Europe. European Planning Studies, 7(6), 693-697.

UNESCO (2013). ISCED Fields of Education and Training (ISCED-F 2013): Manual to accompany the International Standard Classification of Education 2011. 